\title{
Why the Expansion of the Universe Appears to Accelerate
}

\author{
Paul Smeulders \\ 3 The Nursery, Sutton Courtenay, OX14 4UA, UK \\ Email: paul.smeulders@btinternet.com
}

Received March 30, 2013; revised April 29, 2013; accepted May 24, 2013

Copyright (c) 2013 Paul Smeulders. This is an open access article distributed under the Creative Commons Attribution License, which permits unrestricted use, distribution, and reproduction in any medium, provided the original work is properly cited.

\begin{abstract}
A Speed of Light falling over time inversely proportional to the expansion of the Universe leads to an experimentally observed exponential changing of the Red Shift over time. It is necessary to re-define the Angular Impulse Momentum in order to get a consistent expansion of space on all levels. Conservation of Energy and this newly defined Angular Impulse Momentum then leads to the requirement that all clocks slow down in time inversely proportional to the Red Shift, independent of whether the Speed of Light is constant or not. From the Lorentz equation it then follows that Expansion occurs over space-time and not over space alone. A steady state expansion in true time is then transformed into an exponential expansion for an observer with a local clock. A finite lifetime of the Universe is transformed to an infinite lifetime for these observers including elementary particles.
\end{abstract}

Keywords: Acceleration of Expansion; Expansion of Space-Time; Exponential Expansion; Conservation Laws; Dark Energy

\section{Introduction}

It has been shown that Speed of Light measurements are basically flawed and that therefore the actual Speed of Light can never be measured correctly [1]. The actual Speed of Light can therefore vary over time without us being able to observe this directly. It has been shown that the Hubble Law fits the Supernovae data [2] in an excellent way when a varying Speed of light is taken into account. This then automatically leads to a universe with an expansion that is exponentially in time.

\section{The Red Shift}

With a Speed of Light changing over time, the red shift is a combination of the contribution by the expansion of the universe with scale $a(t)$ and the contribution of the change in the speed of light $c(t)$. It has been shown that the red shift becomes:

$$
z+1=\frac{\lambda_{o b s}}{\lambda_{e m}} \frac{\lambda_{e m}}{\lambda_{0}}=\frac{\lambda_{o b s}}{\lambda_{0}}=\frac{c(t)}{c_{0} a(t)}
$$

The first ratio of the wavelengths is due to the expansion of the universe and the second part related to the change in the speed of light.

Figure 1 shows the measured distance of the supernovae versus the product of the Speed of Light $c(t)$ and the Red Shift $z$. Here the Speed of light has been assumed to vary inversely proportional to the expansion rate of the Universe $a(t)$. The measured distance of the supernovae has therefore the following relation to the red shift $z$ :

$$
D_{L}=c_{0} t_{0} \sqrt{z+1}[(z+1)-1]
$$

This should be compared with the calculated one [1]:

$$
D_{\text {now }}=\frac{1}{a(t)} \int_{t}^{t_{0}} \frac{c(t)}{a(t)} \mathrm{d} t=\frac{c_{0} t_{0}}{a(t)} \int_{x}^{1}(z+1) \mathrm{d} x
$$

with $x=t / t_{0}$. Equating Equations (2) and (3) and

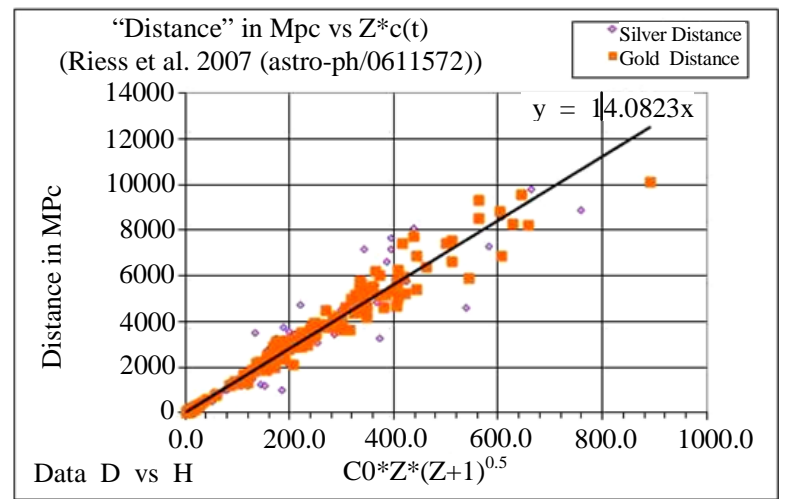

Figure 1. The measured distance $D_{L}$ versus the product $c(t)$. $\mathrm{z}$ in which $c(t)=c_{0} / a(t) . c_{0}$ is the speed of light at present. A remarkable good fit with the data is obtained with a characteristic time $t_{0}$ of 14.082 Gyear. 
eliminating $c_{0} \cdot t_{0}$, one gets:

$$
\int_{x}^{1}(z+1) \mathrm{d} x=z \cdot(z+1)^{\gamma+\frac{1}{2}}
$$

if one stipulates that $a(t)=(z+1)^{\gamma}$. By varying $\gamma$ one can get a range of $a(t)$ and $c(t)$ pairs that satisfy equation (1) and lead to the observed modified Hubble Law. For instance $\gamma=-1$ represents the expanding universe with a constant speed of light. All other values of $\gamma$ lead to a varying speed of light over time. The nature of the red shift limits the range of gamma to values of: $-1 \leq \gamma \leq 0$. A universe that would not expand at all is represented by $\gamma=0$.

It has been shown [1] that the following relation exist between the Red Shift $z$ and the time $x=t / t_{0}$ as function of gamma $\gamma$ :

$$
\begin{aligned}
1-x & =\left(\gamma+\frac{3}{2}\right) \frac{(z+1)^{\gamma+\frac{1}{2}}}{\gamma+\frac{1}{2}} \\
& -\left(\gamma+\frac{1}{2}\right) \frac{(z+1)^{\gamma-\frac{1}{2}}}{\gamma-\frac{1}{2}}+\frac{1}{\gamma^{2}-\frac{1}{4}}
\end{aligned}
$$

And for $\gamma \approx-\frac{1}{2}$ we have $1-x \approx \ln (z+1)$ with a relative error of $\left(\gamma+\frac{1}{2}\right) \ln (z+1)$.

We may therefore conclude that the solution is quasi-exponential for a range of values of gamma: $-0.8<\gamma<0.3$ and for Red shifts of up to $z=10$. This is consistent with the observation that the expansion of the universe seems to accelerate in recent times.

The Red Shift is:

$$
z+1 \approx \mathrm{e}^{-\frac{t}{t_{0}}} \text { with } a(t)=\mathrm{e}^{-\frac{\gamma t}{t_{0}}} \text { and } c(t)=c_{0} \mathrm{e}^{-\frac{(1+\gamma) t}{t_{0}}}
$$

The value of gamma $\gamma$ determines the relation between the expansion scaling $a(t)$ and the speed of light $c(t)$. The time $t=0$ is the present time, $t_{0}=14$ Gyear and $c_{0}$ is the speed of light in our, the present time.

\section{The Expansion and Conservation Laws}

It is has been shown in [1] that for a planet of mass $m$ orbiting a star with mass $M$ at a distance $\mathrm{r}$ in an expanding universe energy conservation is readily obtained. And that with changing $a(t)$ and $c(t)$ energy is conserved if the ratio of $v / c$ is conserved. This implies too that relativistic energies and equations are conserved or unchanged.

It was also shown that the standard law of angular impulse momentum m.xㅗ can not be correct, since it leads to conflicting dependences of $r(t)$. A simple modification of the definition of the angular impulse momentum eliminates this problem and then $r(t)=a(t) \cdot r_{0}$.

The correct definition of the Angular Impulse Momentum is:

$$
\boldsymbol{P}=m c^{2}\left[\frac{\boldsymbol{v}}{c} \times \frac{\boldsymbol{r}}{a}\right]
$$

This momentum is conserved when the universe expands with $r(t)=a(t) \cdot r_{0}$. It is clear that this definition is the same as the one we are used to in our time when $a(t)=1$.

However the clocks all change now with the expansion and the clock frequency changes with the Red Shift:

$$
\omega(t)=\omega_{0}(z+1)
$$

The Angular Impulse Momentum applies also for the hydrogen atom, which equals:

$$
m_{e} \cdot v \cdot a_{0}(t) \cdot \frac{c(t)}{c_{0} a(t)}=\hbar
$$

With this definition of the angular impulse momentum, conservation implies that the scaling with the expansion scale factor $a(t)$ is maintained for both planet orbits and atoms.

Another important detail is that the electric charge is changing with the expansion:

$$
e^{2}(t)=e^{2}\left(t_{0}\right) \cdot \frac{a(t) c_{0}}{c(t)}=e^{2}\left(t_{0}\right) \frac{1}{z+1}
$$

The dependence of the clocks on the Red Shift has implications for the Lorentz equation and so on Relativity. It can be seen that Relativity as such is unaffected by the expansion of the universe.

The Lorentz equation is:

$$
\begin{aligned}
x^{2}+y^{2}+z^{2}-c^{2} t^{2} & =a^{2}(t) \cdot\left(x_{0}^{2}+y_{0}^{2}+z_{0}^{2}\right) \\
& -\frac{c^{2} c_{0}^{2} a^{2}(t)}{c^{2}} t_{0}^{2}
\end{aligned}
$$

This just states that the Lorentz length, like all lengths, too is multiplied by $a(t)$.

Also Quantum mechanics are unaffected. It can readily be seen that all energy levels of the Hydrogen atom are in units of $m c^{2} \alpha^{2}$, which is independent of $a(t)$. Without this the analysis of the Redshift would have been meaningless.

\section{Time Expansion}

The expansion of the Lorentz length shown above leads to an important conclusion: namely that the expansion of the Universe does not just occur over space but over space-time. And that causes an observation of a phe- 
nomenon that evolves linearly in true time to be observed on an apparent exponential time scale for an observer with a clock depending on $a(t)$.

Assume that an observer clock runs with time $t$. That time is a function of the true time: $d t=(z+1) \cdot d \tau$. The inverse of the Red Shift is zero at the time of the Big Bang and unity at the present time. Now assume that this latter function evolves linearly in the true time:

$$
\frac{\mathrm{d}}{\mathrm{d} \tau}(z+1)^{-1}=\frac{1}{t_{0}} .
$$

One then gets for the time derivative in the observer time:

$$
\frac{1}{(z+1)^{-1}} \frac{\mathrm{d}}{\mathrm{d} t}(z+1)^{-1}=\frac{1}{\tau_{0}}
$$

It can readily be seen that this yields: $z+1=\mathrm{e}^{\frac{-t}{t_{0}}}$.

Together with $a(t)=\mathrm{e}^{-\frac{\gamma t}{t_{0}}}$ and $c(t)=c_{0} \mathrm{e}^{-\frac{(1+\gamma) t}{t_{0}}}$ and $t_{0}$ $=\tau_{0}$. It can be concluded that an observed exponential behaviour of the Red Shift over time is related to a steady state expansion of the universe in true time. There is no real acceleration and therefore there is no need for Dark Energy. The exponential expansion is an artefact of the expansion of space-time.

\section{The Relation between $a(t)$ and $c(t)$ and Scaling with $z+1$}

The value of gamma $\gamma$ controls the relationship between $a(t)$ and $c(t)$.

The size of the objects seen depends on the value of gamma and these are scaling with $(z+1)^{\gamma}$. On the other hand the angular rotation of objects scale with just $(z+$ 1).

There exists tempting supporting data for the exponential scaling of the expansion. It has been shown that the galaxy size (in kpc) is a function of the red-shift $z$ [3]. The data in Figure 2 show that the size of galaxies scale as $(1+z)^{\gamma}$, with $\gamma$ equal $0,-1$ and -2 . For $\gamma=-0.5$ the galaxies would scale as the scale factor of the expanding universe, namely as $a(z)=(1+z)^{-0.5}$. But other values of $\gamma$ around $-1 / 2$, like $-2 / 3$ are also certainly possible. The latter leads to the condition that $c^{2} \cdot a$ is constant [4]. Another value of $\gamma=-1 / 3$ yields $c \cdot a^{2}$ being constant and leads to a much stronger inflation. This may be seen in Figure 3 which shows the evolution in true time of the scale factor $a(\tau)$.

\section{Discussion}

The exponential expansion now comes out of the expansion of space-time and this is consistent with observations of the Supernovae distances. It is independent of whether the Speed of Light is constant over time or not.

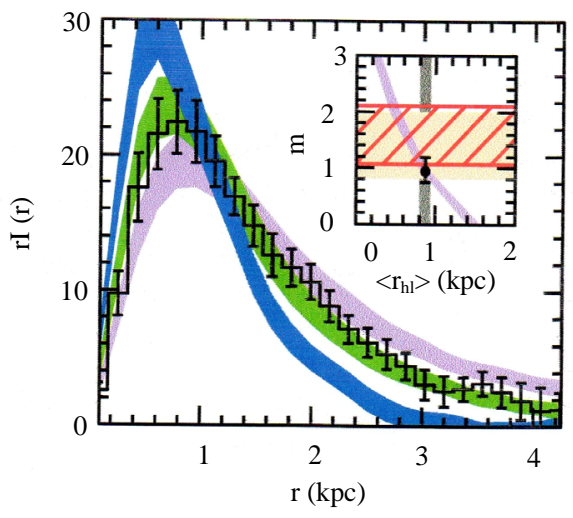

Figure 2. Courtesy of R. Bouwens [3]. The radial luminescence versus radius in kpc for various scaling's of the size of the galaxies versus $z+1$. Namely : $(z+1)^{-2},(z+1)^{-1}$ and $(z+$ $1)^{0}$. Clearly the correct exponent is between -1 and 0 .

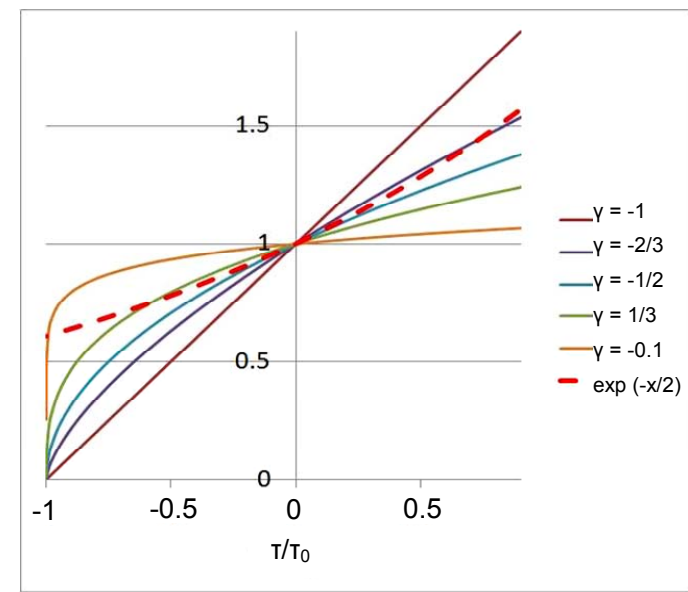

Figure 3. The scale factor a of the universe versus true time $\tau / \tau_{0}$ for various values of $\gamma, \tau_{0}$ is Gyear. The closer $\gamma$ is to zero the more inflation occurs at the time of the Big Bang. The red broken line shows the exponential scaling of the $a(t)$ for the observer time $t$ and $\gamma=-1 / 2$.

There is still a degree of freedom of how the expansion scales with the Red Shift. It is clear that $a(t)$ scales with $(z+1)^{\gamma}$ with $\gamma$ around $-1 / 2$, but a $\gamma$ of $-2 / 3$ or $-1 / 3$ cannot be excluded at all for the moment. The scaling of the Speed of Light over time is tied up to the scaling of the expansion with the Red Shift. To narrow the possible range of $\gamma$, even more accurate measurements of the size of galaxies will have to be made or physics models will have to determine the scaling.

\section{Conclusion}

The exponential scaling law gives a very good match to the Supernovae observations. A re-definition of the Angular Impulse Momentum is necessary in order to get a consistent expansion of the Universe. This leads to an expansion of space-time and not just space alone. This latter on its turn transforms a steady state expansion into 
for an observer exponential expansion, which explains quite well the observed acceleration of the expansion. The change in the clock rate applies to observers as well as elementary particles alike. So that all things large and small must have recycled many times over since the start of the Universe.

\section{Acknowledgements}

The author would like to thank Dr. A. Riess for making his data available in his paper. Also he would like to thank Dr. R. Bouwens for the use of his figure on the scaling of the galaxy size with the Red Shift, which gives considerable support for the exponential expansion. Finally he would like to express his gratitude for the con- tinued support by Prof. A. Kavokin of the Mediterranean Institute of Fundamental Physics.

\section{REFERENCES}

[1] P. Smeulders, Journal of Modern Physics, Vol. 3, 2012, pp. 345-349. doi:10.4236/jmp.2012.34047

[2] A. Riess, et al., Astrophysical Journal, Vol. 659, 2007, pp. 98-121. doi:10.1086/510378

[3] R. Bouwens, et al., Astrophysical Journal, Vol. 611, 2004, pp. L1-L4. doi:10.1086/423786

[4] A. Alfonso-Faus, "Variación de las Constantes Físicas con el Tiempo Cosmológico,” Instituto de Astrofísica de Canarias, XII Edición de Los Encuentros Relativistas Españoles (ERE), Tenerife, 1987. 